NANOSYSTEMS: PHYSICS, CHEMISTRY, MATHEMATICS, 2015, 6 (5), P. 680-688

\title{
Encapsulation of iron atoms between framgments of graphene planes
}

\author{
${ }^{1}$ A. V. Siklitskaya, ${ }^{2}$ V. I. Ivanov-Omskii, ${ }^{2}$ M. S. Chekulaev, ${ }^{2}$ S. G. Yastrebov, ${ }^{3}$ R. Smith, \\ ${ }^{4}$ A. Slav, ${ }^{4}$ C. Morosanu, ${ }^{5}$ S. V. Kozyrev \\ ${ }^{1}$ Institute of Theoretical Physics, University of Warsaw, Warsaw, Poland \\ 2 Ioffe Institute, St.Petersburg, Russia \\ ${ }^{3}$ Loughborough University, Department of Mathematical Sciences, Loughborough, \\ United Kingdom \\ ${ }^{4}$ National Institute of Materials Physics, Bucharest, Romania \\ ${ }^{5}$ Center for advanced studies, Peter the Great St.Petersburg Polytechnic University, \\ St.Petersburg, Russia \\ Yastrebov@mail.ioffe.ru, SVKozyrev@spbcas.ru
}

PACS 81.05.ue

DOI 10.17586/2220-8054-2015-6-5-680-688

In this paper, we apply results of analysis of the Raman spectrum from an amorphous carbon film modified with iron $(a-C: F e)$ which leads to construction of clusters containing fragments of graphene and a layer of iron atoms. Such clusters may be candidates for the role of microwave radiation absorbers. For the Raman experiments we performed deposition of a thin film of $a-C: F e$. Details of the film deposition process, together with the corresponding Raman experiments are presented in this paper. Comparison with a literature model was performed for the intensity ratio of the maxima for specific Raman bands, using the letter $D$ to represent "disorder" and the letter $G$ to represent "graphite". Comparison gives evidence for the existence of nanosize fragments of graphene embedded in an amorphous matrix of the $a-C: F e$ film. The diameter of the fragments is predicted to attain a value of about $1.2 \mathrm{~nm}$. Moreover, in a comparison with experimental data for defective graphite, the position of the maximum of the $D$ band for $a-C: F e$ appeared to be red shifted. This could support the proposition that damping of Raman-active oscillations occurs by an electron gas in fragments of graphene after introduction of iron, e.g. after intercalation. On the basis of these estimates, we modeled a symmetrical polycyclic aromatic hydrocarbon having a similar size and converted it into fragment of a graphene plane by removal of hydrogen atoms occupying the edge states. To preserve symmetry, we placed atoms of iron on top of the fragment, situating them exactly above the centers of hexagons at a certain distance and placed another fragment of graphene on the top of the iron atoms, symmetrically. For a distance of 2.52 Ảbetween the centers of the hexagon and the iron atom, distortions of carbon-carbon valence bonds and angles were found to be minimal, as shown through optimization of the system's geometry using the Avogadro molecular editor. This result supports an hypothesis of graphene fragments during the growth of an amorphous carbon film modified by simultaneous addition of a dopant metal such as iron. This example may illustrate the stability of a two-dimensional electron gas confined between the fragments.

Keywords: graphene, iron, intercalation, carbon, nanoclusters, encapsulation.

Received: 1 October 2015 


\section{Introduction}

Academic interest has recently been growing for the relatively new nanocomposites formed from clusters of graphene and metals. For example, two dimensional inclusions of iron were incorporated between planar fragments of graphene due to the lattice parameter match between graphene and two-dimensional iron $[1,2]$. On the other hand, routine technological methods to achieve similar goals have so far not been reported. Simultaneous magnetron sputtering of graphite and metal targets may be one way in which such clusters may be formed. In this case, a stacking between two dimensional metal and graphene plane fragments may occur, usually termed intercalation. The metal atoms can be situated either between two graphene planes or above a single fragment $[3,4]$. However, experimental evidence for the existence of such fragments is limited to exploiting arguments concerning the activation of $D$ and $G$ Raman bands in infrared spectra of the composites [3-7]. The authors of these papers term the incorporation of metal atoms in a carbon environment as 'encapsulation'. For the case of pairs of graphene planes and iron metal atoms fragments placed between them, one may consider these planes as outer "shells" of a capsule and use this term. However, there has until now been no direct observations of the intercalated graphene clusters, although some modelling results have been published $[3,4]$.

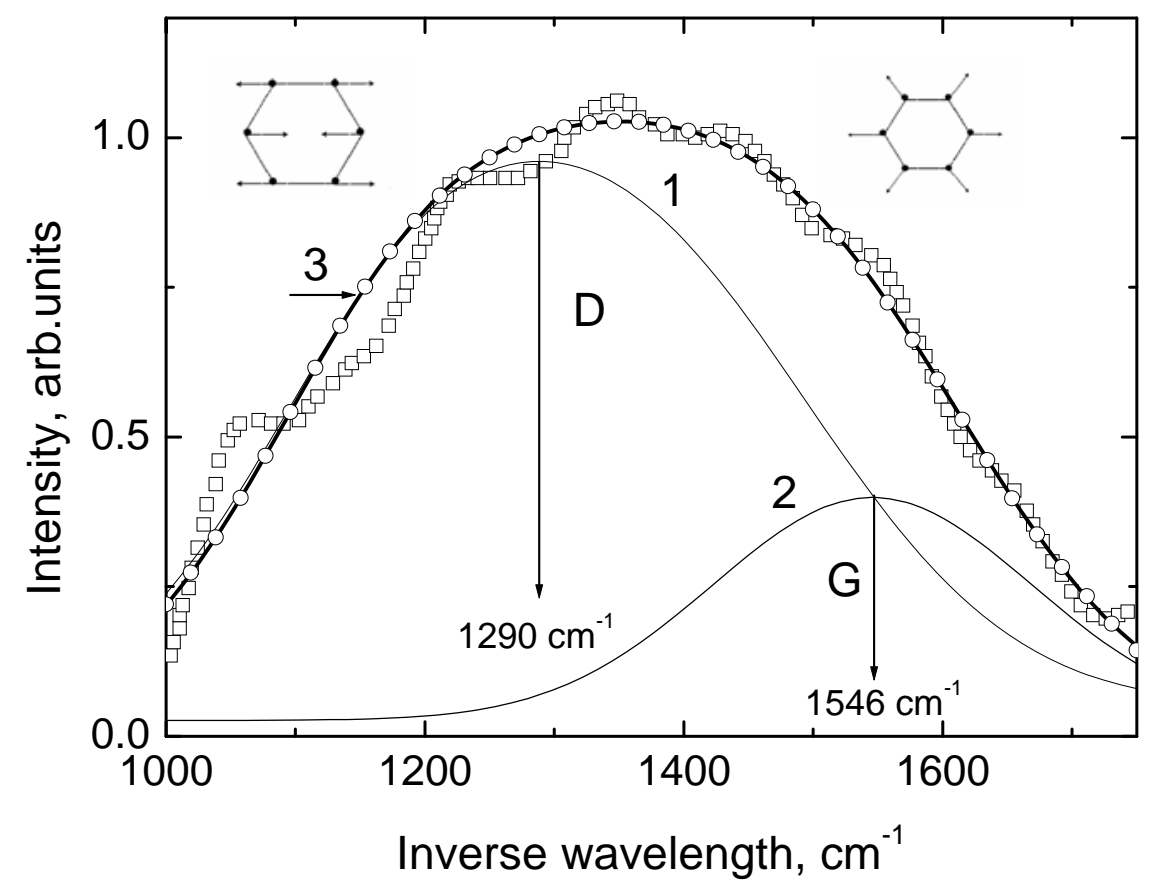

FIG. 1. Raman spectrum of a thin film of a-:Fe $([\mathrm{Fe}] /[\mathrm{C}]=0.62)$ and its Gaussian decomposition: empty squares stand for experimental data. Insets show two types of oscillations of carbon atoms in a hexagon contributing to $D$ and $G$ Raman bands, correspondingly. An interference pattern is distinctly recognizable. Result of smoothing [17] is presented by empty circles. Solid lines depict Gaussian decomposition discriminating $D$ and $G$ bands as contour 1 and (contour 2), correspondingly; curve 3 portrays the sum of contours 1 and 2 


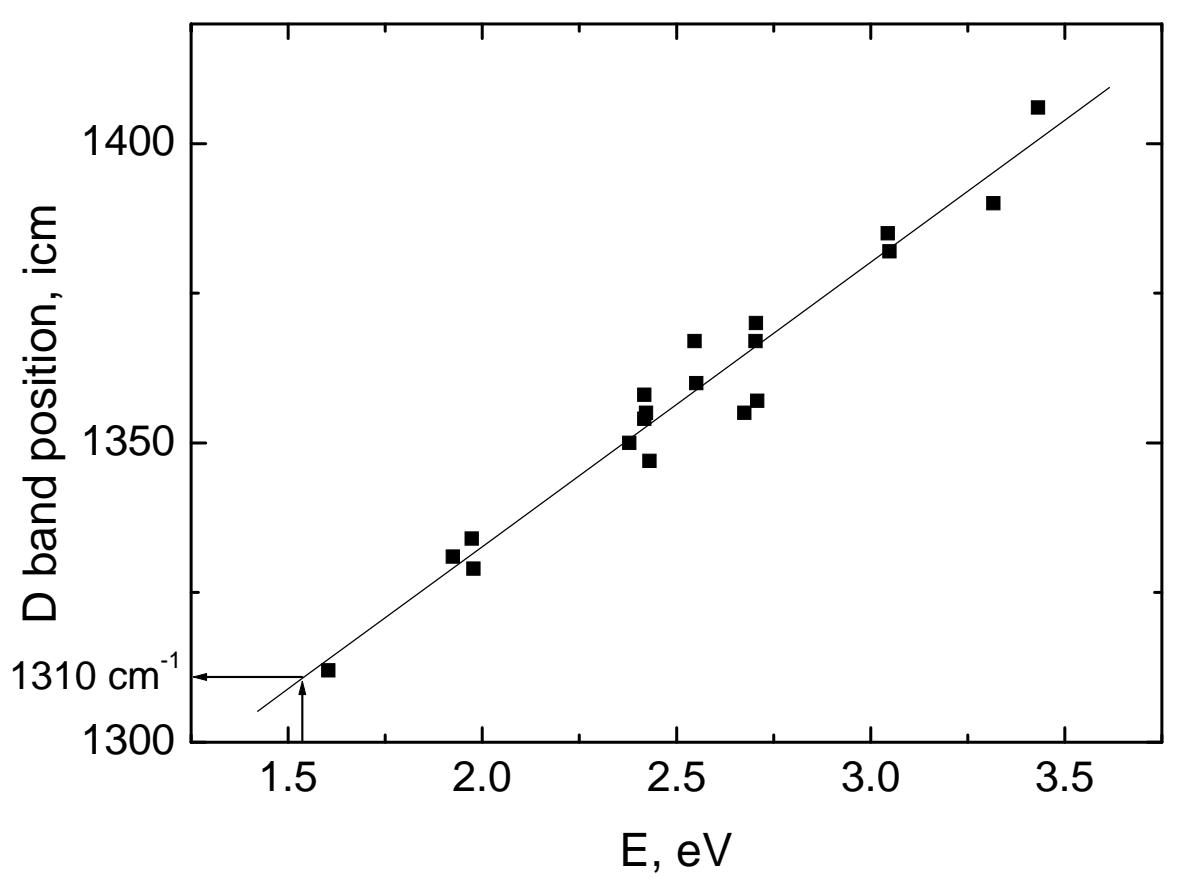

FIG. 2. Dependence of the spectral position of the Raman $D$ band for defective graphite as a function of energy of photons exciting the Raman spectra, [18]. Arrows depict the position of the $D$ band for the photon energy used in this paper

Practical interest in amorphous carbon containing encapsulated clusters of magnetic metals was linked to the intriguing possibility of electromagnetic radiation absorption in the microwave spectral region (frequency $\sim 10 \mathrm{GHz}$ ) [8]. This could provide opportunities to use nanocomposite films for purposes of electromagnetic shielding in many different areas, including stealth technology [9]. This effect might be linked to the excitation of Frölich-like resonance because of either the specific magnetic properties of nanosize portions of ferromagnetic materials [10] or their special dielectric characteristics. Among valuable magnetic properties, hysteresis losses play the most important role for contribution to the absorption of microwaves. However, for magnetic clusters of several nanometers in size, the hysteresis losses are negligibly small [11]. Thus, the observed effect of microwave absorption might be simply explained by the peculiarities of electromagnetic absorption by particles of special shapes. These could be e.g., flat nanoclusters confining a two-dimensional electron gas embedded in the nanocomposite because the Frólich resonance shifts for this particular case to the microwave region (see, e.g. [12]). It is natural to postulate the existence of twodimensional conductive clusters that may absorb microwave radiation and real fragments of graphene. Thus, in this study, we investigate fragments of graphene and ordered layers of iron atoms. If a single layer of iron is absorbed by a single fragment of graphene, one may term this situation as decoration. When two or more fragments are involved, this situation might be termed as intercalation. For symmetry reasons, we present the case of intercalation when a layer of iron atoms is placed between a pair of fragments of graphene. To be precise, iron atoms are situated above the centers of the carbon hexagons. A similar geometrical picture was considered [13], but more fragments of graphene planes were considered there. 


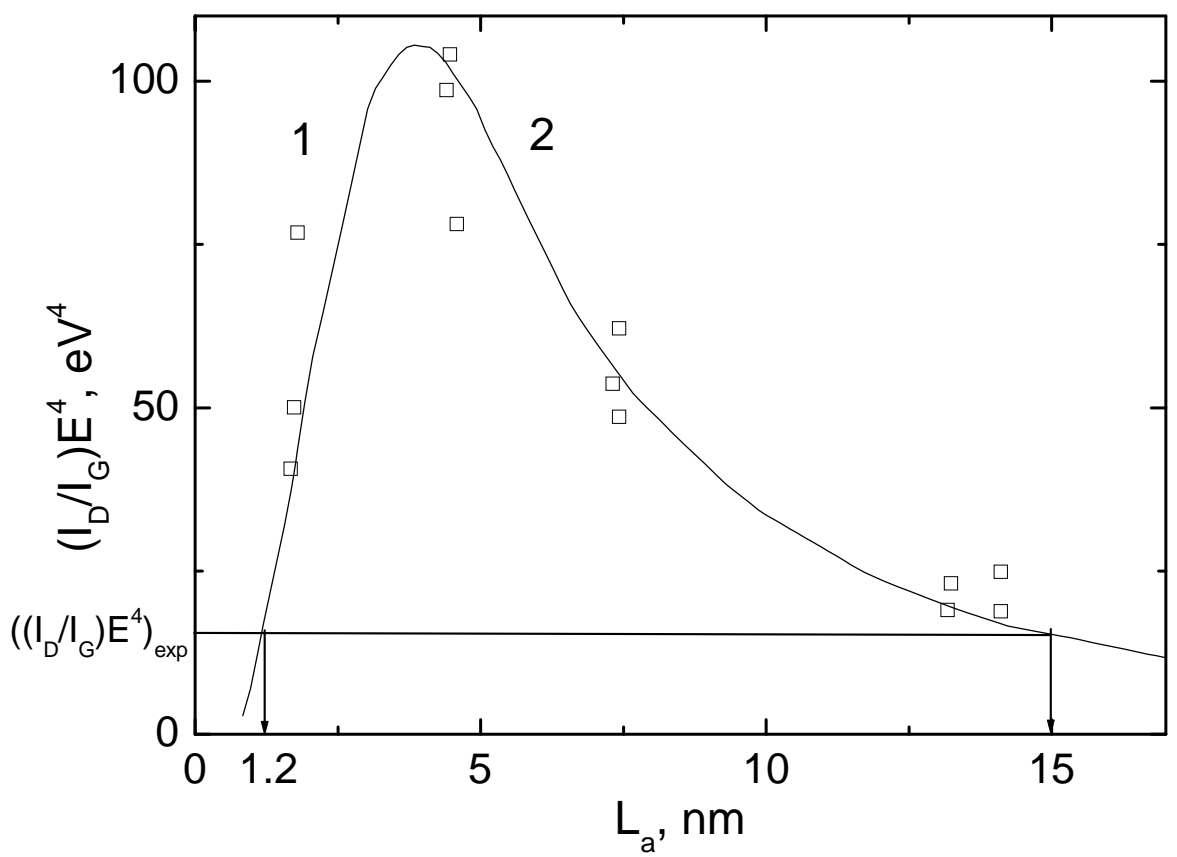

FIG. 3. Dependence of the ratio $\frac{I_{D}}{I_{G}} E^{4}$ on the distance $L_{a}$ between defects in exfoliated graphene exposed to ion bombardment. The left hand part of the curve to the maximum represents fragmented graphene embedded in the amorphous matrix; points to the right from the maximum characterize passage through the percolation threshold; here $I_{D}$ and $I_{G}$ are intensities of Raman scattering measured at the maxima of the contours of $D$ and $G$ correspondingly; $E$ is the energy of the photon used for excitation of the Raman spectrum. Empty squares depict experimental data from [14], [15]. The solid line shows the best fit to the equation in [15]. The left arrow gives the size of graphene fragments for the case of an amorphous film of a-C :Fe: $\left(\frac{I_{D}}{I_{G}} E^{4}\right)_{\exp }$ as determined from Fig.1. The right arrow shows the hypothetical case when the percolation cluster consists of linking fragments of graphene
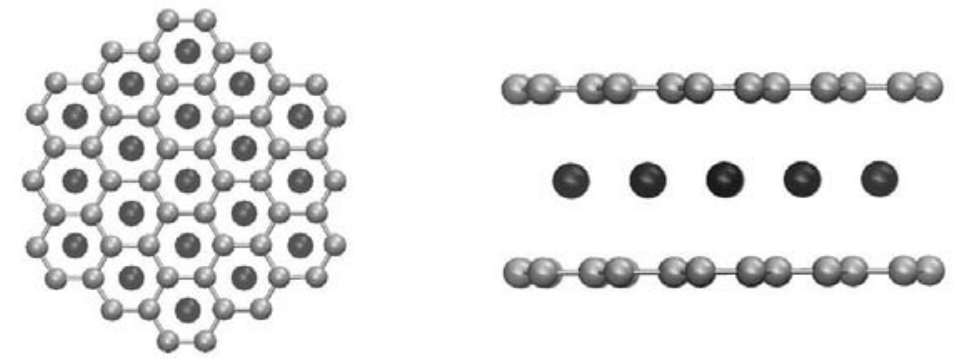

FIG. 4. A view of a pair of fragments of graphene planes encapsulating a layer of iron atoms after structure optimization performed by the Avogadro package. Gray and dark gray circles portray atoms of carbon and iron correspondingly 


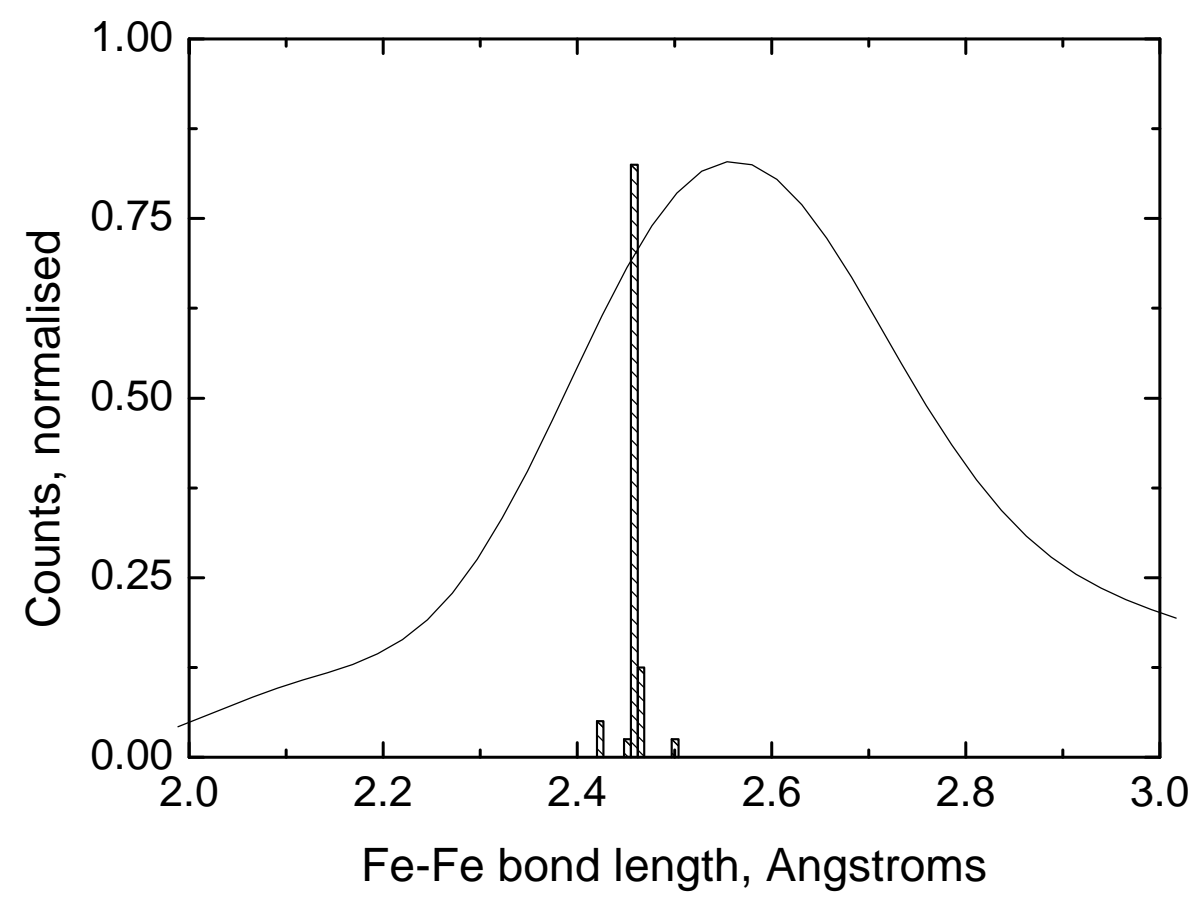

FIG. 5. The Fe bond angle distribution in the graphene layers. Bars represent the Fe-Fe interatomic distance after optimizing the geometry of the atomic configuration as in Fig. 4. The full line corresponds to part of the radial distribution function for amorphous iron [20]

Here we derive geometric parameters of the cluster by comparison of the ratio of specific Raman bands obtained from our experimental data and calibration curve suggested recently in $([14],[15])$. The geometric optimization of the model of the cluster was performed using the Avogadro molecule editor [16]. This is useful for qualitative investigation of the stability of new materials. The statistical distributions of bond length between atoms forming the cluster are also presented and discussed here.

\section{Experimental}

The film was grown by the simultaneous sputtering of graphite and iron with an alternating current magnetron system operating at a frequency of $1.78 \mathrm{MHz}$. More technical details are presented in [11]. Here we note that the chemical composition of film, thickness and specific density were obtained using both Rutherford backscattering and nuclear reactions with a deuteron beam of energy $1 \mathrm{MeV}$. Analysis of the experimental data shows a concentration ratio $[\mathrm{Fe}] /[\mathrm{C}]$ of 0.62 and traces of oxygen with a corresponding ratio $[O] /[C]$ of 0.05 . The film was $\sim 1 \mu \mathrm{m}$ in thickness and the density $\varrho=3.9 \mathrm{~g} / \mathrm{cm}^{3}$. Raman spectra were obtained using an Ocean Optics R-2001 spectrometer operating at $785 \mathrm{~nm}$ and $500 \mathrm{~mW}$ radiation. The radiation was delivered to the investigating sample by a fiber waveguide. A similar waveguide was used for detection of the scattered radiation. 


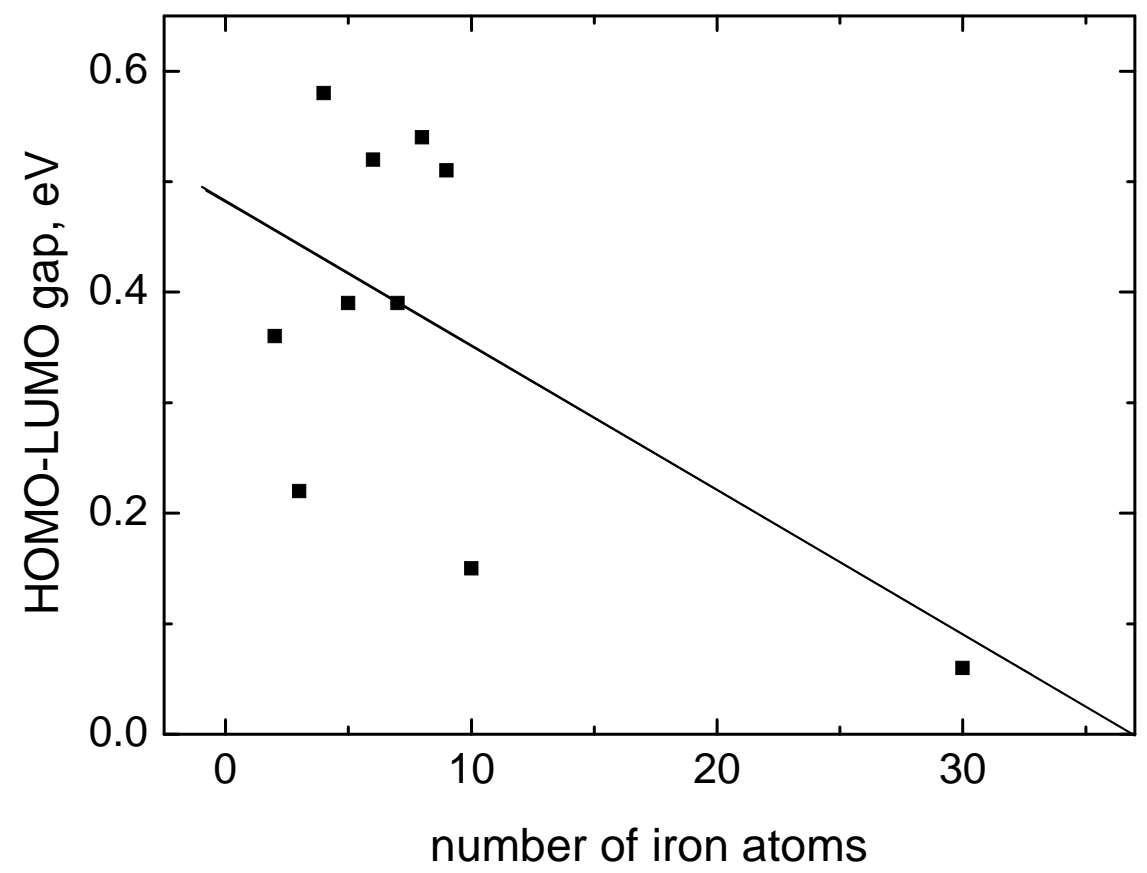

Fig. 6. Dependence of the HOMO-LUMO gap as a function of the number of atoms in the iron nanocluster [21], [22]. The gap becomes zero for $\mathrm{N}=37$

\section{Results and discussion}

\subsection{Geometrical characteristics of graphene fragment}

Figure 1 shows the Raman spectrum for $a-C: F e$ in the area corresponding to atomic vibrations of the carbon matrix where specific bands $D$ and $G$ manifest themselves. The spectrum looks like a wide band slightly modified with an interference pattern. We applied the Swanepoel method [8] for smoothing the interference and the result is presented in Fig. 1 by empty circles. The decomposition of the resulting function was performed using the best fit by a sum of two Gaussians extracting the contribution of $D$ and $G$ lines from the background. A comparison was performed between the spectral position of the Raman band $D$ extracted from the experiment presented in Fig.1 and the dispersion dependence for the position of the $D$ band for disordered graphite depicted in Fig.2. The dependence presented in Fig.2 is plotted for the position of the $D$ band measured in inverse wavelength versus the exciting Raman spectra photon energies. The arrow in Fig.2 shows the prediction for the position of the $D$ band for our experiment, equal to $\sim 1310 \mathrm{~cm}^{-1}$. However, the Gaussian decomposition of data presented in Fig.1, gives a different inverse wavelength value, $1290 \mathrm{~cm}^{-1}$. It means that the position of the $D$ band presented in Fig. 1 is red-shifted in comparison with the prediction. That might be used as evidence of a damping process of the $C-C$ vibrations that might be realized through excitation of a free electron gas in the vicinity of the conducting cluster. This fact may be used as evidence for the existence of conductive inclusions embedded in an amorphous carbon matrix. Among them, the irondecorated or iron-intercalated fragments of graphene might occur. Note that the ratio of $\frac{I_{D}}{I_{G}}$ characterizes the size of the graphene fragment forming the amorphous carbon matrix, 
as was demonstrated in recent papers [14], [15] where a correction to the energy of photons exciting the Raman spectrum $E$ was performed. Here $I_{D}$ and $I_{G}$ are the intensities for Raman scattering measured at the maxima of the contours of $D$ and $G$ correspondingly. To be precise, the calibration curve from [15] establishing a link between the ratio and distance between defects in exfoliated graphene is depicted in Fig.3. This dependence consists of two parts: that of the left side of the maximum and that of the right part. The right part corresponds to the case when a percolation cluster containing linking graphene fragments has formed. The left part characterizes an ensemble of graphene fragments separated from each other by an amorphous matrix. Therefore, a direct comparison of the experimental the value obtained from Fig.1, $\left(\frac{I_{D}}{I_{G}} E^{4}\right)_{e x p}$, with the dependence presented in Fig.3 by a solid line, is valid. The left arrow in Fig. 3 corresponds to the case of an amorphous structure and gives an estimation for the graphene fragment size for our sample of $\approx 1.2 \mathrm{~nm}$. The right arrow corresponds to the hypothetical case of formation of the infinite percolation cluster in an amorphous carbon matrix.

\subsection{Optimisation of geometry}

Based on the above information for the size of a fragment of graphene, we chose as a model a polycyclic aromatic hydrocarbon $C_{54} H_{18}$ [19] having almost the same size as predicted by Raman spectroscopy. We made a slight modification with the hydrocarbon, removing atoms of hydrogen occupying the edge states. Afterwards we constructed the layered system. The iron atoms were placed exactly above the centers of each hexagon belonging to a basal fragment of graphene maintaining a certain distance $L$ above the plane. Another fragment of graphene was placed symmetrically on the top of the layer of iron atoms keeping the same distance from the each hexagon center to each iron atom of $L$. Thus, we investigate case of "intercalation", but one may reduce the system size to obtain results for the case "decoration" without loss of generality.

Different occupations of empty places above hexagons may occur in reality. Here, we considered maximal occupation of empty places by analogy with previous paper where we modeled the interaction of cobalt with unsymmetrical graphene fragments.

A schematic view of the carbon-iron cluster after optimization of the geometry by Avogadro is portrayed in Fig.4. Note that after optimization both the Fe-Fe and C-C bond lengths only slightly deviate from their initial values of $2.61 \AA$ and $1.42 \AA$ respectively. It is seen in the Figure 5 that the interplanar distances are slightly changed after optimization, deviating from the initial value of $L=2.52 \AA$. It might be interesting to exploit ab initio modeling to investigate details of the electronic structure of carbon-iron clusters more exactly before a firm conclusion on the possibility of absorption of microwaves by the clusters can be formed. However, for ab initio modeling, computation time is expensive. Thus, we analyze here the electronic spectrum qualitatively. First of all it is important to estimate with more details the degree of amorphization of the iron layer obtained after optimization of the geometry because electronic properties are very sensitive to this parameter. For this purpose, we present in Fig.5 an histogram of the Fe-Fe interatomic distribution in comparison with a similar distribution for amorphous iron. One may see in Fig.5 that the distribution of Fe-Fe bonds is very sharp in comparison with amorphous iron itself.

Furthermore, since we are dealing with nano-dimensional clusters, it is interesting to see how the effects of electron spectrum size quantization can affect the electrical conductivity of the iron layer and adjacent graphene plane fragment.

Figure 6 shows the dependence of the energy gap between the free and occupied states in iron nanoclusters (HOMO-LUMO gap), as a function of the number of atoms 
forming them. It is seen that the dependence may be approximated by a linear trend that reaches zero when the number of atoms is approximately equal to $M=37$. In this case, the cluster of iron is metallic. Note that this cluster will differ only slightly in size from the analog, shown in Figure 4. This may indicate a good estimation of the size of the cluster size performed by analyzing the Raman spectrum. Because a possible difference may exist between the work functions of the graphene plane fragments and two-dimensional layer of iron metal, electrons can be either localized in the vicinity of iron layer or may delocalize within a whole fragment of graphene -iron - fragment of the graphene sandwich.

When electrons in an iron cluster behave as they do in a bulk metal, one may use a value known for a bulk iron: 4.5-4.7 eV [23]. In turn, to evaluate the work function $W$ of a fragment of graphene one can use the expression: $W=I_{p}+\frac{E_{g}}{2}$, where $I_{p}$ is ionization potential for the fragment and $E_{g}$ is its width of optical gap.

The value of $I_{p}$ might be estimated using a well-known equation for polycyclic aromatic hydrocarbons: $I_{p}=5.1+0.7 E_{g}(\mathrm{eV})[24]$. For a fragment of graphene containing 37 hexagonal rings ( 37 is number of atoms when the layer of iron becomes conductive), the value for $E_{g}$ might be estimated using an approximate equation for symmetric hydrocarbons [25]: $E_{g}=2|\beta| M^{-1 / 2}$; here $2|\beta| \approx 2.9 \mathrm{eV}$ is the energy of interaction between pair of adjacent $\pi$ orbitals. $M$ is number of hexagons in the fragment. $E_{g} \approx 0.95 \mathrm{eV}$ for $M=37$, thus $W=$ $6.24 \mathrm{eV}$. That is the work function for a fragment of graphene supporting a conductive iron layer is bigger than that for bulk iron. One may expect for this situation some smearing of the free electron gas from the iron layer to the graphene fragments.

\section{Conclusions}

The estimations performed in this paper lead to two preliminary conclusions:

1. Flat particles containing fragments of graphene planes separated by a layer of iron may be present in an a-C:Fe structure.

2. Whether the fragment would be metallic or not will depend upon the possibility of the formation of the iron layer with dimensions that are sufficient for suppression of quantum effects.

The paper was presented at the 10th Simposium "Thermodynamics and Material Science" 7-11.09.2015, St. Petersburg, Russia.

\section{References}

[1] Kosugi K., Bushiri M. J., Nishi N. Formation of air stable carbon-skinned iron nanocrystals from $\mathrm{FeC}_{2}$. Appl. Phys. Lett, 2004, 84, P. 1753-55.

[2] Zhao J., Deng Q., Bachmatiuk A., et al. Free-Standing Single-Atom-Thick Iron Membranes Suspended in Graphene Pores. Science, 2014, 343, P. 1228-32.

[3] Ivanov-Omskii V. I., Smorgonskaya E. A. Charge displacement induced by intercalation of graphite-like nanoclusters in amorphous carbon with copper. Physics of the Solid State, 1999, 41(3), P. 786-88.

[4] Ivanov-Omskii V. I., Smorgonskaya E. A. Modification of the electron spectrum and vibrational properties of amorphous carbon by copper doping. Semiconductors, 1998, 32(8), P. 831-37.

[5] Ivanov-Omskii V. I., Zvonareva T. K., Frolova G. S. Vibration modes of carbon in hydrogenated amorphous carbon modified with copper. Semiconductors, 2000, 34(12), P. 1391-96.

[6] Zvonareva T. K., Ivanova E. I., Frolova G. S. et al. Vibratonal spectroscopy of $a C: H(C o)$. Semiconductors, 2002, 36(6), P. 695-00.

[7] Ivanov-Omskii V. I., Frolova G. S. IR spectroscopy of copper-intercalated graphite nanoclusters in amorphous carbon. Tech. Phys. Lett., 2000, 26(7), P. 623-24.

[8] Lutsev L. V., Yakovlev S. V., Zvonareva T. K. et al. Microwave properties of granular amorphous carbon films with cobalt nanoparticles. J. Appl. Phys., 2005, 97, P. 104327/1-6. 
[9] Alexeev A. G., Shtager E. A., Kozyrev S. V. Physical Principles of Stealth Technology. St.Petersburg.: VVM, 2007. 283 p.

[10] Draine B. T., Lazarian A. Magnetic dipole microwave emission from dust grains. Astrophys. J., 1999, 512, P. 740-54.

[11] Yastrebov S. G, Ivanov-Omskii V.I., Pop V, et al. Magnetic properties of iron-modified amorphous carbon. Semiconductors, 2005, 39(7), P. 840-44.

[12] Bohren C. F., Huffman D. R. Absorption and Scattering of Light by Small Particles. Weinheim.: WileyVCH Verlag GmbH, 1998, 545 p.

[13] Belenkov E. A. Formation of Graphite Structure in Carbon Crystallites. Inorganic Materials, 2001, 37(9), P. 928-34.

[14] Lucchese M. M., Stavale F, Ferreira Martins E. H., Vilani C. et al Quantifying ion-induced defects and Raman relaxation length in graphene. Carbon, 2010, 4(8), P. 1592-97.

[15] Cancado L. G., Jorio A., Ferreira Martins E.H. et. al. Quantifying Defects in Graphene via Raman Spectroscopy at Different Excitation Energies. Nanoletters, 2011, 11(8), P. 3190-3196.

[16] Hanwell M. D., Curtis D.E., Lonie D.C., et al. Avogadro: An advanced semantic chemical editor, visualization, and analysis platform. J Cheminform, 2012, 4(1), P. 1-17.

[17] Swanepoel R. Determination of the thickness and optical constants of amorphous silicon. J. Phps. E: Sci. Instrum., 1983, 16, P. 1214-33.

[18] Ferrari A. C. Raman spectroscopy of graphene and graphite: Disorder, electron-phonon coupling, doping and nonadiabatic effects. Solid State Communications, 2007, 143, P. 47-57

[19] Alberty R., A., Chung M., B., Reif A.K. Standard Chemical Thermodynamic properties of Polycyclic Aromatic Hydrocarbons and their Isomer Groups. III. Naphthocoronene Series, Ovalene Series, and First Members of Some Higher Series. Phys Chem Ref Data, 1990, 19(2), P. 350-70.

[20] Yamamoto R., Shibuta H., Doyama M. Computer simulation of radiation damage in amorphous metals. J.Nucl.Mater., 1979, 85-86, P. 603-06.

[21] Wang L., Hudson G. A. Advanced Molecular Dynamics Simulations on the Formation of Transition Metal Nanoparticles. In: Molecular Dynamics - Theoretical Developments and Applications in Nanotechnology and Energy; Ed. Lichang Wang L.- INTECH Open Access Publisher, 2012, 424 p.

[22] Cervantes-Salguero K., Seminario J. M. Structure and energetics of small iron clusters. J. Mol. Model., 2012, 18, P. 4043-52.

[23] Hölzl J., Schulte F. K. Work function of metals In Solid Surface Physics. Springer Tracts in Modern Physics, 2006, 85, P. 1-150

[24] Kuroda H. Ionisation potential of polyciclic aromatic hydrocarbons. Nature, 1964, P. 1214-15.

[25] Robertson J., O'Reilly E. P. Electronic and atomic structure of amorphous carbon. Phys.Rev.B, 1987, 35(6), P. 2946-57. 